\title{
Surface Roughness Studies with DALLAS-Detector Array for Laser Light Angular Scattering
}

\author{
T. V. Vorburger, E. C. Teague, F. E. Scire, M. J. McLay, and D. E. Gilsinn \\ National Bureau of Standards, Washington, DC 20234
}

\begin{abstract}
Accepted: October 14, 1983
An instrument has been developed to study surface roughness by measuring the angular distributions of scattered light. In our instrument, a beam from a He-Ne laser illuminates the surface at an angle of incidence which may be varied. The scattered light distribution is detected by an array of 87 fiber optic sensors positioned in a semicircular yoke which can be rotated about its axis so that the scattered radiation may be sampled over an entire hemisphere. The output from the detector array is digitized, stored, and analyzed in a laboratory computer. The initial experiments have concentrated on measurements of stainless steel surfaces which are highly two-dimensional and which yield scattering distributions that are localized in the plane of incidence. The results are analyzed by comparing the angular scattering data with theoretical angular scattering distributions computed from digitized roughness profiles measured by a stylus instrument. The theoretical distributions are calculated by. substituting the roughness profiles into the operand of an integral equation for electromagnetic scattering developed by Beckmann and Spizzichino. This approach directly tests the accuracy of the basic optical theory.
\end{abstract}

Key words: angular distribution; diffraction; diffuse scattering; electromagnetic scattering; fiber optics; light scattering; optical fiber; optical scattering; roughness; surface roughness; texture; topography.

\section{Introduction}

Optical scattering techniques have been used for a long time to monitor the surface roughness of industrial parts ranging from crankshaft bearings $[1]^{1}$ to $\mathrm{x}$-ray mirror prototypes [2]. These techniques lend themselves to on-line surface inspection in industry because they are intrinsically area-averaging, highspeed methods. A single measurement can yield a quantity that is closely related to some average property of the surface roughness [3]. However, optical scattering methods are almost exclusively used in an empirical way because the quantitative deduction

\begin{abstract}
About the Authors, Paper: T. V. Vorburger, E. C. Teague, F. E. Scire, M. J. McLay, and D. E. Gilsinn are with the Mechanical Production Metrology Division ir NBS' Center for Manufacturing Engineering. The work on which they report was supported by NASA and the NBS Office of Nondestructive Evaluation.
\end{abstract}

\footnotetext{
${ }^{1}$ Figures in brackets indicate literature references at the end of this paper.
}

of roughness parameters from optical measurements is extremely difficult because of the complexity of optical scattering itself. Empirical approaches [1,4] have been developed which rely on the use of a number of calibration surfaces with known roughness parameters that are similar to the unknown surfaces to be measured. These calibration standards enable the operator to calibrate the surface measuring instrument empirically. Although this comparator approach is effective, we attempt here to derive optical scattering quantities from more basic principles. Then perhaps, optical methods could be applied to surface roughness problems more generally and with a higher degree of confidence.

This difficulty of understanding is particularly acute for engineering surfaces where the roughness heights are typically in the range between 0.1 and $1.0 \mu \mathrm{m}$. $\mathrm{He}-\mathrm{Ne}$ lasers with wavelength $\lambda=0.6328 \mu \mathrm{m}$ are commonly used in such applications because of their relative safety, good stability, ease of alignment, and other features. However, this means that the roughness heights are on the same order of magnitude as the wavelength of light [5]. The mathematical description is much more complicated in this regime 
than it is for optically smooth surfaces $[6,7]$ where the effect of surface roughness is a small perturbation on the basic phenomenon of specular optical scattering, i.e., where the surface basically functions like a mirror.

The present work is an attempt to develop a better mathematical description of optical scattering phenomena for engineering surfaces. The ultimate goal of this work is an optical scattering apparatus for reliable and routine measurements of roughness parameters without resorting to specially fabricated comparator standards.

After a brief experimental overview in section 2, we discuss the apparatus in detail in section 3. There follows an outline of the theory in section 4 . Section 5 deals with the experimental results, and section 6 (Analysis) compares the experimental and theoretical scattering distributions. In section 7 we discuss the limitations of the present work as well as previous work and probable future directions. Some experimental notes are included as an appendix.

\section{Experimental Overview}

When a beam of laser light is reflected by a rough surface, the radiation is scattered into an angular distribution (fig. 1) according to the laws of physical optics. The intensity and the pattern of the scattered radiation depend on the roughness heights, the roughness spatial wavelengths, and the wavelength of the light [6-8]. In general, small spatial wavelength components diffract the light into large angles relative to the specular direction, and long spatial wavelength components diffract the light into small angles. Most surfaces have a broad range of spatial wavelengths,

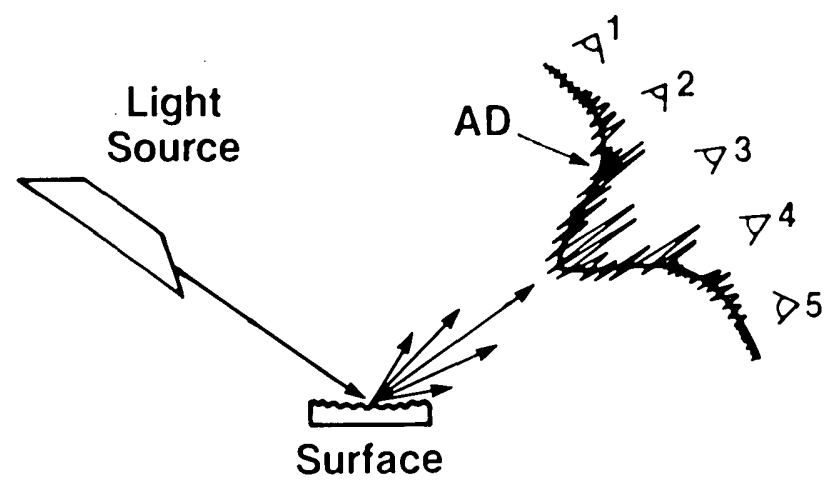

Figure 1-Schematic diagram of a laser beam scattered by a rough surface. The pattern consists of the overall angular distribution envelope (AD) and a fine structure known as speckle. A simple optical detection system is also shown. Detector 3 measures the intensity in the specular direction. Detectors $1,2,4,5$ measure other components of the angular distribution. and the light is therefore diffracted over a range of angles.

For very smooth surfaces, most of the reflected light propagates in the specular direction. As the roughness increases, the intensity of the specular beam decreases while the diffracted radiation increases in intensity and becomes more diffuse. In addition, the angular distribution of diffuse radiation consists of a fine grainy structure called speckle [9], which shows up as intensity contrast between neighboring points in the scattered field. Finally, the light wave may undergo a change in its polarization state upon reflection from the surface.

In this work, we study how the angular distribution is related to the detailed topography of engineering surfaces. In particular we explore the following fundamental question: If the detailed surface topography were perfectly known, could the angular scattering distribution be predicted from available optical scattering theories? If so, that basic knowledge might lead to optical techniques for measuring the roughness of surfaces without resorting to calibration artifacts. If one cannot relate optical scattering to surface roughness in this very straightforward way, then it is likely that metrologists will be limited to empirical approaches for the characterization of engineering surfaces by optical scattering.

Our approach uses an optical instrument called DALLAS (detector array for laser light angular scattering), a stylus profiling instrument interfaced to a minicomputer for accurate characterization of surface topography, and a fairly elementary optical scattering theory. Surface profiles measured by the stylus instrument are substituted into the scattering theory to generate angular distributions which may be compared with those directly measured by DALLAS for the same surfaces. We report here some preliminary results with this equipment.

\section{Apparatus}

A block diagram of the twofold apparatus is shown in figure 2. In the DALLAS experiment, a beam of laser light illuminates the rough surface under test and the scattered radiation is collected by an array of 87 detectors. The signals are sequentially routed by a scanner to a digital voltmeter which functions as an analog-to-digital converter. The resulting angular distribution is stored in a desktop microcomputer and may be compared with distributions generated from the stylus experiment. In the latter system, a commercial stylus instrument measures surface profiles and stores them on a magnetic disk on a large 

paratus. The optical experiment, DALLAS, is shown at the top, and the stylus system, at the bottom. Experimental and theoretical angular distribudesktop microcomputer shown near the center.
Figure 2-Block diagram of the aptions may be compared on the

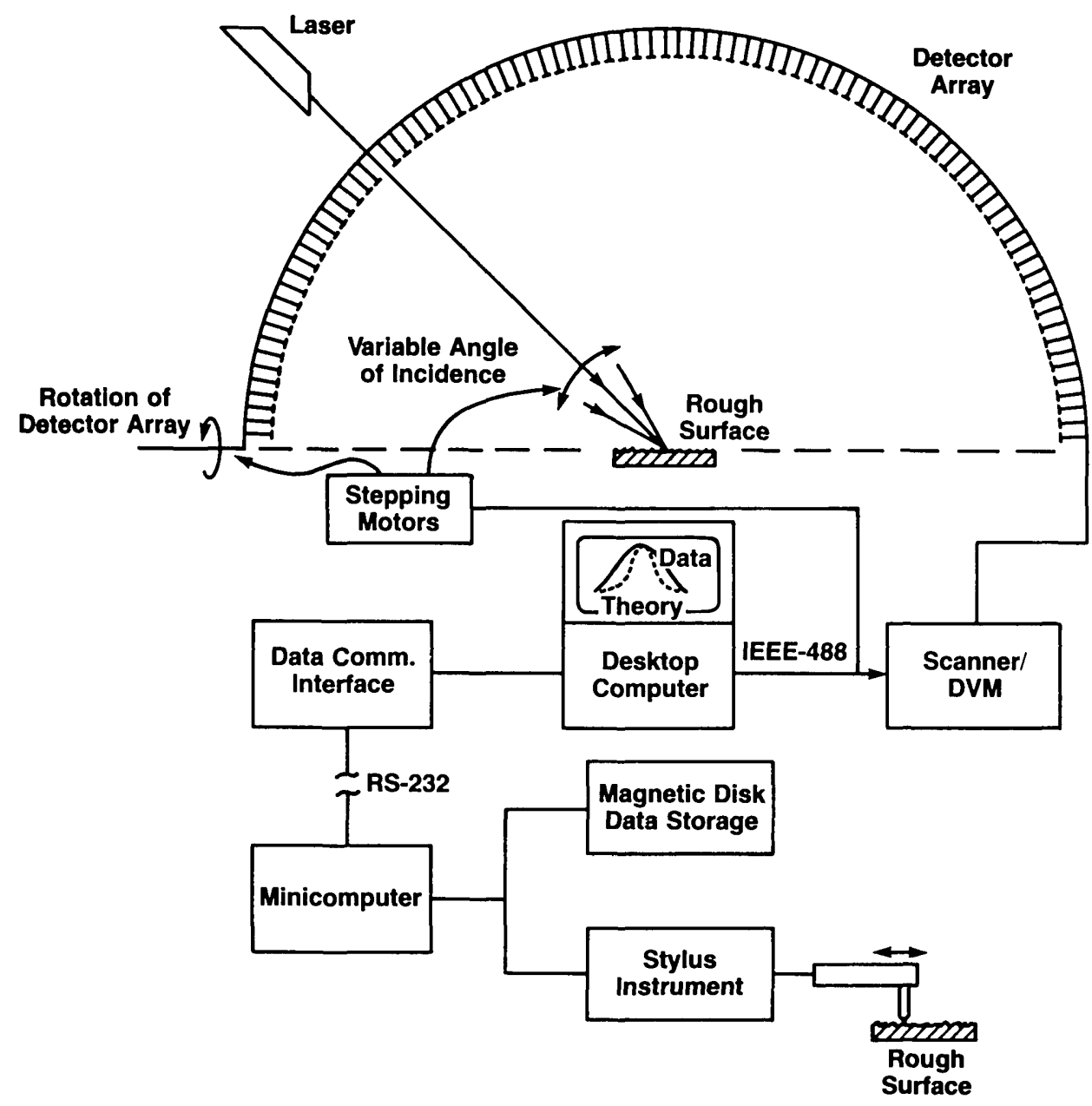

minicomputer. Optical scattering distributions are then calculated from these profiles and the results may be transmitted to the microcomputer by a hardwired RS232 interface.

\subsection{Optical Apparatus}

The apparatus for measuring intensity distributions as a function of scattering angle from surfaces is shown in figure 3 and consists of an illumination system and a detection system.

The illumination system consists of a $5 \mathrm{~mW} \mathrm{He}-\mathrm{Ne}$ laser with linear polarization, a quarter-wave plate to produce circular polarization, an automatic shutter, and a rotating assembly of two mirrors, M1 and M2, to direct the laser beam onto the specimen surface. The angle of incidence may be varied by a stepping motor which controls the angular position of M1 and M2. The illuminated region of the specimen is a spot approximately $2 \mathrm{~mm} \times 3 \mathrm{~mm}$, depending on the angle of incidence. The detection system consists of an array of 87 detectors spaced $2^{\circ}$ apart in a semicircular yoke (diam $=164 \mathrm{~mm}$ ) which is centered on the illumination spot on the specimen. The yoke can be rotated about one axis by a stepping motor so that the detectors can sample practically the entire hemisphere of radiation scattered from the surface.

Each detector consists of a lens, an optical fiber, and a PIN Si photodiode with an integral op-amp circuit. Each lens has a diameter of $4.4 \mathrm{~mm}$ and subtends an angle of about $1.5^{\circ}$ in the yoke. It collects the radiation and focuses it onto the fiber which transmits the radiation to the photodiode. The output voltage signals from the op-amps are scanned by a 100-channel scanner, digitized, and stored in the desktop microcomputer using BASIC language software. At present, a single angular scan of the 87 detectors takes about $10 \mathrm{~s}$ and yields intensity distributions which span over 5 orders of magnitude in intensity. That is, the rms noise of the apparatus is approximately $50 \mu \mathrm{V}$, and the saturation voltage of the detectors is about 9 V. The nonlinearity of two typical detectors was measured by comparing their voltage outputs with that of a highly linear, standard Si detector. Over a 


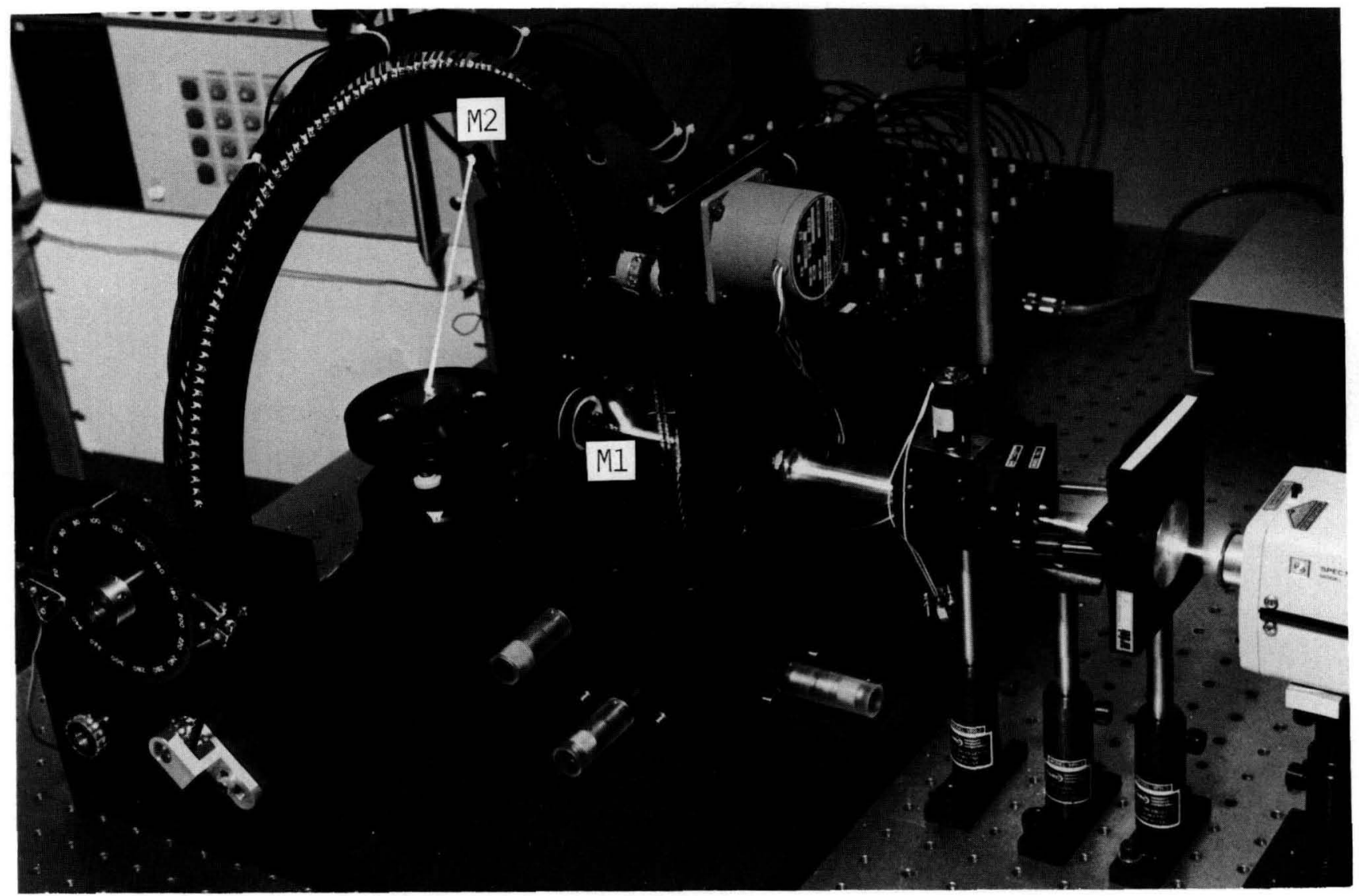

Figure 3-DALLAS in operation. Mirrors (M1 and M2) direct laser beam onto the surface of the specimen located under semicircular yoke supporting the detection system.

dynamic range of $10^{5}$ in input light intensity, the nonlinearity of the output voltage was less than $2 \%$ or $50 \mu \mathrm{V}$, whichever is greater. The relative linearity of the 87 detectors with light intensity (tracking) has also been checked. Over $31 / 2$ orders of magnitude of light intensity, the output voltages track one another with a standard deviation of $2 \%$ or 2.5 times the rms noise, whichever is greater.

The 87-point angular distributions may be stored permanently on magnetic tape cassettes or plotted on the CRT of the microcomputer for comparison with the angular scattering calculations predicted from stylus data. Additional notes on the detection system are given in section 8 .

\subsection{Stylus Apparatus}

The stylus system has been described previously [10-12]. It consists of a Talystep ${ }^{2}$ stylus instrument

\footnotetext{
${ }^{2}$ Certain kinds of commercial equipment are identified in this article to specify adequately the experimental procedure. In no case does such identification imply recommendation or endorsement by the National Bureau of Standards, nor does it imply that the equipment identified is necessarily the best available for the purpose.
}

interfaced to a minicomputer. As the stylus traverses the peaks and valleys of the surface, the vertical motion is converted to a time-varying electrical signal which undergoes 12-bit A/D conversion. The result is a roughness profile consisting of 4000 digitized points that may be permanently stored on the magnetic disk. The horizontal length of the profile is approximately $1.84 \mathrm{~mm}$, and the point spacing is $0.46 \mu \mathrm{m}$. The horizontal resolution of the instrument is approximately $1 \mu \mathrm{m}$, limited by the high frequency falloff of the stylus response function.

The ultimate vertical resolution of the stylus instrument is approximately $0.3 \mathrm{~nm}$ over the length of the stylus profile. The vertical resolution of the digitized profile may also be limited by the quantization increment of the 12-bit A/D converter, which depends on the magnification scale of the stylus instrument controller. For the rougher surfaces, the controller was set at a low magnification; the smallest quantization increment was approximately $1.2 \mathrm{~nm}$.

Each surface was sampled with 10 stylus traces evenly distributed over an area approximately 3 $\mathrm{mm} \times 6 \mathrm{~mm}$. Hence, the total amount of topography 
information amounts to 40,000 digitized points for each surface.

\subsection{Specimens}

A commercial set of four surface specimens [13] was studied with both the stylus and DALLAS techniques. Three of the specimens were specially machined to produce highly two-dimensional roughness specimens; that is, each surface has a fairly random roughness profile in one direction and an essentially smooth profile in the perpendicular direction. The fourth specimen was very smooth in all directions on the surface. The two-dimensional nature of the three rougher specimens was quite important.

The specimens were oriented in the DALLAS apparatus so that the roughness direction was in the plane of incidence of the light; therefore, essentially all of the scattered light was in the plane of incidence as well. This arrangement has two beneficial effects: 1) all of the scattered light may be detected by a single scan of the detectors without having to rotate the yoke, and 2) the complex, vector electromagnetic scattering problem reduces to a scalar problem [14-16]. Therefore, the use of these specimens reduces a three-dimensional problem that is both theoretically and experimentally complex to a two-dimensional problem without any approximation. The basic approximations of the optical scattering theory may be tested in a fairly straightforward way.

\section{Theory}

The formulas used to predict the angular scattering distributions involve a basic scalar theory of light scattering which has been investigated by Beckmann and Spizzichino [14] as well as others $[17,18]$. The theory assumes that a plane wave of uniform intensity illuminates the specimen surface and that the electric field on the surface and its normal derivative can be expressed in terms of a surface reflection coefficient [14] independent of the local surface topography. The geometry of this scattering problem is shown in figure 4. The surface is assumed to be two dimensional, i.e., rough in the $x$ direction and smooth in the $y$ direction. The incoming plane wave is represented by the wave vector $\mathbf{K}_{i}$ with angle of incidence $\theta_{i}$ with respect to the normal vector $\mathbf{n}$ of the mean plane of the surface. The functional form for the incident electric field $E_{i}$ is given by $\exp \left(\mathrm{jK}_{i} \bullet \mathbf{r}\right)$. The scattered electric field is to be evaluated for an angle $\theta_{s}$ with corresponding outgoing vector $\mathbf{K}_{s}$. The vector $\mathbf{r}$ extends from some nearby origin 0 to a point on the surface.

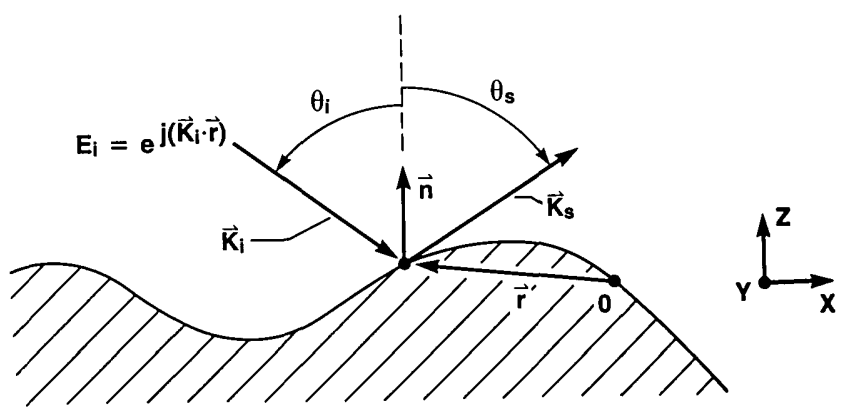

Figure 4-Schematic diagram of the scattering geometry showing the incoming plane wave with wave vector $\vec{K}_{i}$ and angle of incidence $\theta_{i}$, and an outgoing wave vector $\overrightarrow{\mathrm{K}}_{s}$ with scattering angle $\boldsymbol{\theta}_{s} \overrightarrow{\mathrm{r}}$ is the vector from the origin 0 to the point under consideration. (Vector symbols are arrowless [and bold-faced] in the text; arrows are used with such symbols in the caption to match the arrowed symbols of the figure.)

With the foregoing considerations and assumptions, the scattered electric field $E$ can be calculated as a function of scattering angle $\theta_{s}$ in the Fraunhofer zone of the scattered radiation field. It is given by the phase integral over the surface profile $z(x)$ :

$$
E\left(\theta_{s}\right)=C_{0} \frac{\left(1+\cos \left(\theta_{i}-\theta_{s}\right)\right)}{\cos \theta_{i}+\cos \theta_{s}} \int_{0}^{L} \mathrm{e}^{\mathrm{j} \vee \cdot \mathrm{r}} \mathrm{d} x=C_{0} F
$$

where $\mathbf{V}=\mathbf{K}_{i}-\mathbf{K}_{s}, L$ is the length of the illuminated region along the $x$ direction, and $\mathbf{r}=x \hat{\mathbf{i}}+z(x) \hat{\mathbf{k}}$. The vectors $\hat{\mathbf{i}}$ and $\hat{\mathbf{k}}$ are unit vectors in the $x$ and $z$ directions, respectively, and $\mathbf{r}$ contains all of the information concerning the surface profile, and in detail,

$$
\begin{aligned}
\mathbf{V} \cdot \mathbf{r} & =V_{x} x+V_{z} z \\
& =2 \pi / \lambda\left[\left(\sin \theta_{i}+\sin \theta_{s}\right) x+\left(\cos \theta_{i}+\cos \theta_{s}\right) z(x)\right] .
\end{aligned}
$$

The sign convention here is such that $\theta_{s}=-\theta_{i}$ in the specular direction. $C_{\mathrm{o}}$ is a quantity which depends on a number of factors such as $\theta_{i}$ and $E_{i}$, but is independent of $\theta_{s}$. The quantity $F$ contains all of the information concerning the shape of the angular scattering distribution.

The plan of the experiment is as follows: measure a surface profile $z(x)$ point by point, then substitute this profile into the integral, eq (1), to calculate a theoretical angular scattering distribution. This distribution may be compared with the one measured in the DALLAS apparatus for the same surface. In this way the adequacy of the scattering theory can be tested. If the theory is inadequate, then one can remove the various approximations one by one that 
have entered into it and perform the calculation with a more elaborate integral.

\section{Experimental Results}

\subsection{Optical Scattering}

A typical set of angular distribution measurements for one of the four surfaces is shown in figure 5. For all of the surfaces the angle of incidence (AI) was $\pm 30^{\circ}$ with respect to the mean surface normal. We assumed that the mean plane of the surface was the one that gave rise to the specular beam in the angular distribution. This consideration enables one to determine the angle of incidence and the angles of scattering with respect to the mean surface normal in eq (1) if the angle of incidence and scattering angles in the laboratory coordinate system are known. Figure 5 shows pairs of distributions for both $+30^{\circ}$ and $-30^{\circ} \mathrm{AI}$. The difference between the members of a pair is a rotation of the specimen of $180^{\circ}$ about the normal. The deep holes in the distributions occur at the backscattering angle where the mirror M2, which directs the incident light towards the surface, also shadows the detector array from the scattered light. The close match between the members of each pair suggests that there is very little directionality to the roughness peaks and valleys and that the surface is well aligned in the instrument. Two pairs of distributions like these were taken for each of the four specimens.

Distributions for the four specimens are shown in figure 6. These were all taken with an angle of incidence of $+30^{\circ}$. The values given for roughness average $R_{a}$ were calculated from the stylus data (sec. 5.2). The roughness average is defined as the average deviation of the profile about the mean line [19]. There are obvious changes in these distributions as the roughness increases. The $\mathrm{AD}$ for the smoothest surface has a strong specular beam at $\theta_{s}=-30^{\circ}$ and very little scattered light. For $R_{a}=0.20 \mu \mathrm{m}$, the specular beam appears to have vanished but the distribution still peaks strongly at the specular angle. The results for the two roughest surfaces differ significantly from the first two but are quite similar to each another. This is to be expected since at high roughness values $\left(R_{a} \gtrsim \lambda\right)$, the effect on the distribution due to increasing roughness should approach saturation.

\section{PTB Specimen, $0.20 \mu \mathbf{m} \mathbf{R}_{\mathbf{a}}$}

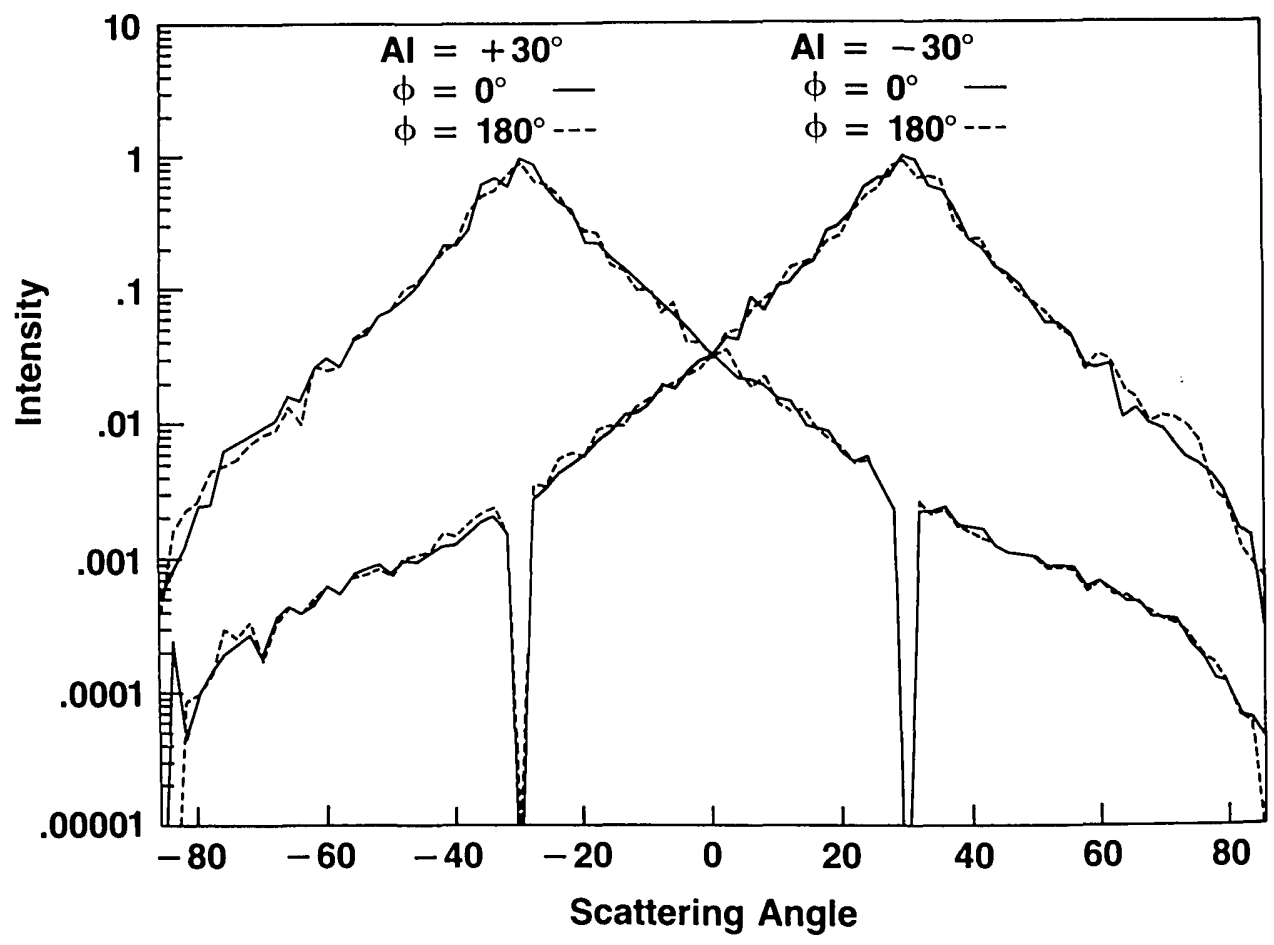

Figure 5-Four angular scattering distributions for a commercial roughness specimen. The measured $R_{a}$ was $0.20 \mu \mathrm{m}$. For each angle of incidence, $\mathrm{AI}=+$ or $-30^{\circ}$, distributions were measured with the specimen oriented at rotation angles $\phi=0^{\circ}$ and $180^{\circ}$ about the normal axis to the specimen. 


\section{PTB Roughness Specimens; AI $=+30^{\circ}, \phi=0^{\circ}$}

Figure 6-Angular scattering data for the set of four commercial roughness specimens. Those with $R_{a}=0.20,0.59$, and $1.6 \mu \mathrm{m}$ are highly two-dimensional, i.e., the surface is essentially smooth perpendicular to the plane of incidence. The angular distributions are normalized so that each has the same total intensity, which is obtained by summing the signals from all the detectors. Note that the intensities are plotted on a logarithmic scale.

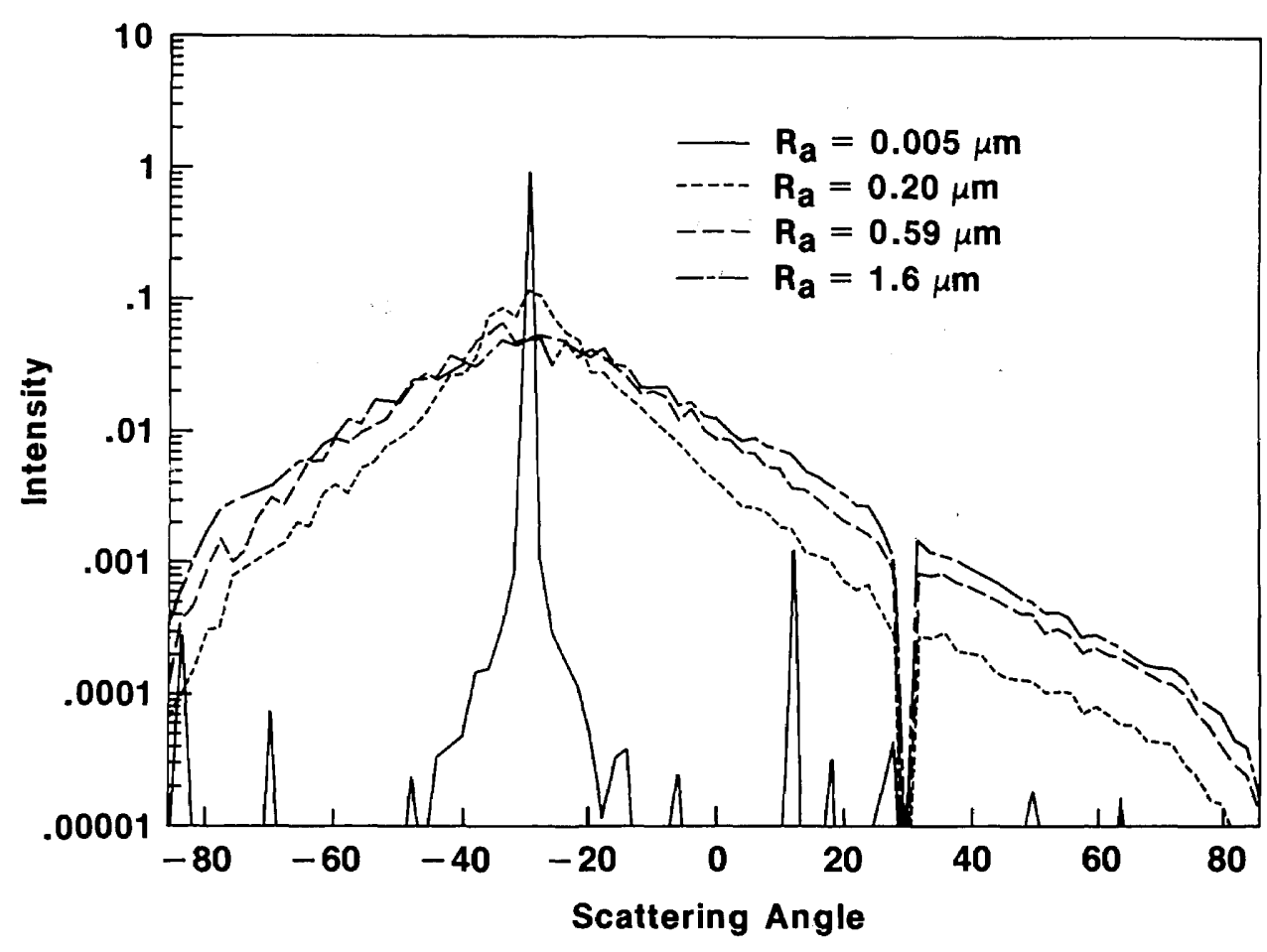

\subsection{Stylus}

Portions of the stylus traces taken for each specimen are shown in figure 7 . The traces were measured without any electronic long-wavelength cutoff, and the profiles are thus undistorted except for the highfrequency cutoff of the instrument and convolution of the profile with the stylus tip, which has a measured width of less than $1 \mu \mathrm{m}$.

The quoted $R_{a}$ values were calculated as the average deviation of the profile around a least squares straight line. For this calculation, the $1.84 \mathrm{~mm}$ trace length was not divided into shorter sampling lengths as is often done in surface metrology. Hence the $R_{a}$ values include effects due to spatial wavelengths limited only by the trace itself.

The three rough specimens were manufactured so that the 2-D "random" roughness pattern repeats itself. This is not evident in the profiles since the periods of the patterns are $1.3,4$, and $4 \mathrm{~mm}$, respectively. Such a periodicity gives rise to very closely spaced diffraction peaks in the angular distribution, but this structure is not resolved by the $1.5^{\circ}$ angular resolution of the detectors. Therefore, the long periodic structure of the surface does not significantly affect the measured angular distributions.

\section{Analysis}

The least squares straight line was subtracted from the stored profile data from the stylus instrument to yield a new digitized profile $z(x)$. It was assumed that the least squares line was equivalent to the $x$-direction of integration in eq (1) and lay in the mean plane that gave rise to the specular beam in the optical experiment.

The profile data $z(x)$ were substituted into eq (1) and the value for the relative field strength $F$ was calculated for each angle $\theta_{s}$. It was not necessary to determine the constant $C_{0}$ to determine the shape of the scattering distribution. The value of $|F|^{2}$ was calculated to derive a quantity proportional to light intensity. This quantity $|F|^{2}$ was then averaged in two ways to develop good statistics in the result:

Speckle Average. Figure 8 shows a close-up view of a segment of the angular distribution projected on the wall of the laboratory. The distribution consists of a complex pattern of fine speckles [9] that vary greatly in intensity from one point to the next. In our apparatus, the average size of the speckles is roughly $0.1 \mathrm{~mm}$ or $0.04^{\circ}[20]$ at the front surfaces of the detector lenses. The lenses themselves span an angle of $1.5^{\circ}$ (about $40 \%$ of the length of figure 8 ); therefore, 


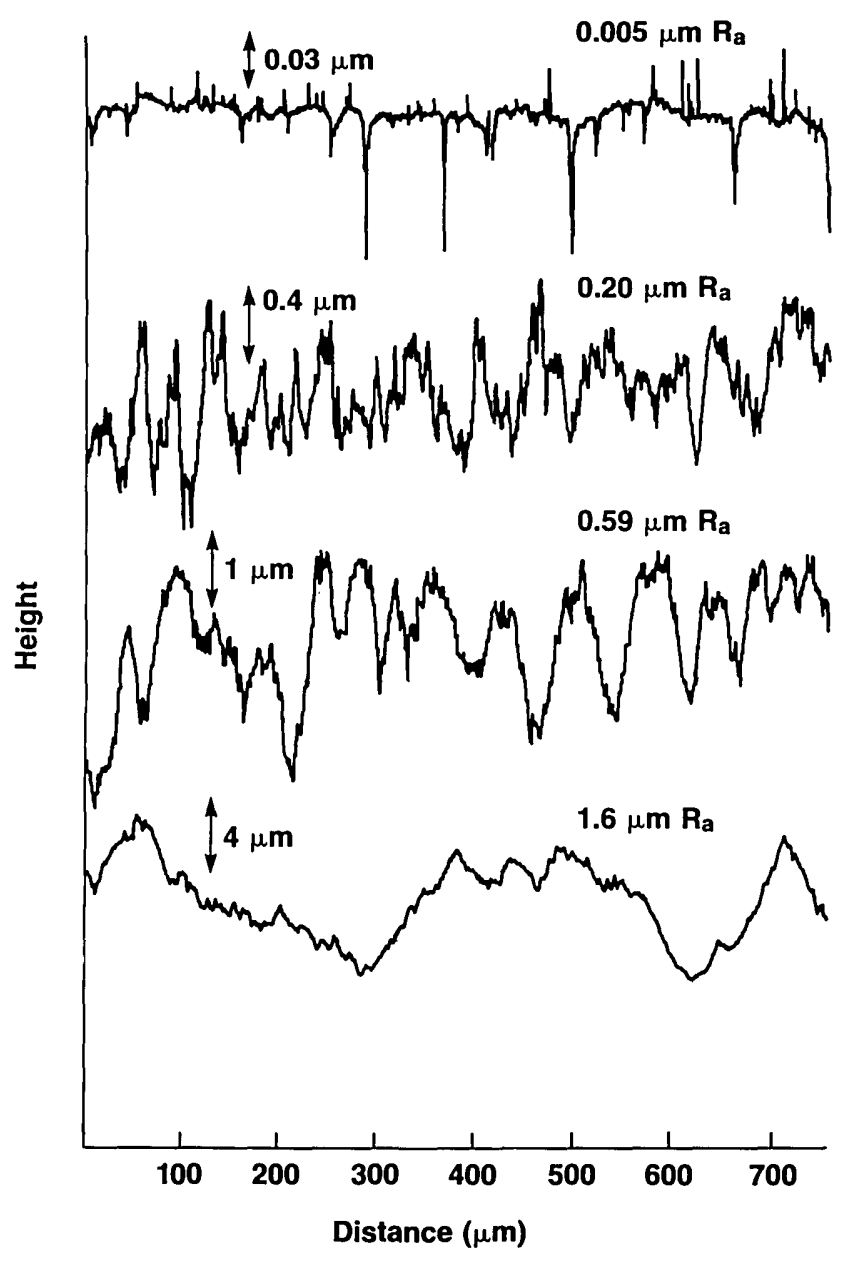

Figure 7-Stylus profiles for the specimens which were studied by the angular scattering measurements shown in figure 6 . Note the differences in the vertical scales.

each detector averages the intensity of a large number of speckles. A single calculation of $|F|^{2}$ from eq (1) would only yield the intensity of a single point. Hence for each detector angle $\theta_{s}$, it is necessary to average over several closely spaced angles to derive reasonable statistics for the overall pattern. In the present experiment, we used seven angles in the plane of incidence separated from one another by $0.05^{\circ}$ and centered about the nominal angle $\theta_{s}$.

Profile Average. The intensity distributions resulting from the speckle average were then averaged over 10 surface profiles in order to achieve some measure of area average which simulates the area averaging of the light scattering approach. In the case of the $1.6 \mu \mathrm{m} R_{a}$ surface, only nine surface profiles were used because we subsequently discovered that one of the profiles had anomalies in the data in several places. To improve the statistics for this case, nine speckle values were calculated instead of seven.

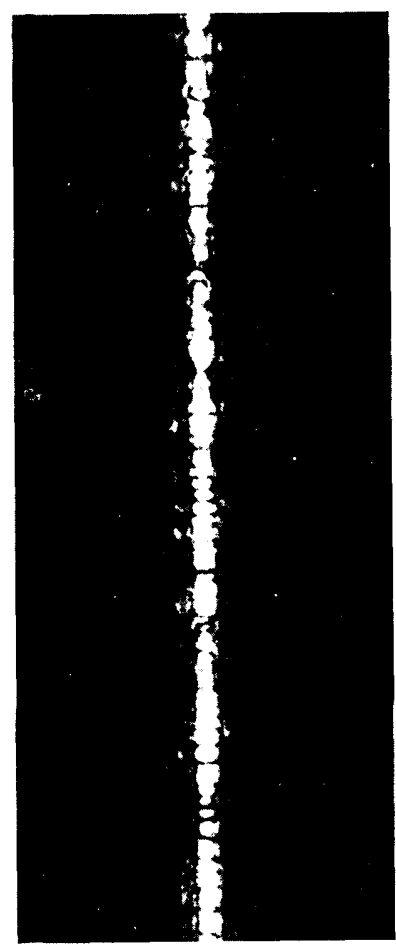

Figure 8-A segment of the angular distribution projected on the wall of the laboratory. The specimen had $R_{a}=0.20$ $\mu \mathrm{m}$. The photograph spans an angle of about $3.5^{\circ}$ from top to bottom. The fine speckle structure is clearly shown.
As a result of the averaging procedure, the relative intensity calculated for each value of $\theta_{s}$ is an average of 70 integrals represented by eq (1) and takes approximately 9 hours on a Perkin Elmer 3230 minicomputer. The resulting distributions are shown by the dotted lines in figures 9-12 and are compared with the measured angular distributions (solid lines). The phase integral calculations successfully reproduce the changes in the experimental distributions from one surface to the next. The specular beam dominates the pattern for the smoothest surface in figure 9. Both the theory and experiment show the same amount of sharp curvature near the specular direction in figure 10 and the same rounded structure in figures 11 and 12 . The major difference between the model and the data is that in all cases, the theoretical distribution falls below the experimental one on the wings. The ratios between the curves are as high as an order of magnitude at some places. Nevertheless, it is gratifying that for these regimes of roughness, the simplified theory can predict much about the distributions.

\section{Discussion}

\subsection{Limitations in the Present Work}

A large number of approximations has entered into the simplified theory of eq (1). Improvements to the preliminary analysis will involve removing each of 
Figure 9-Data vs. calculation for the mirror-like surface with $R_{a}=0.005 \mu \mathrm{m}$. The distributions are normalized in the same way as those in figure 6 .

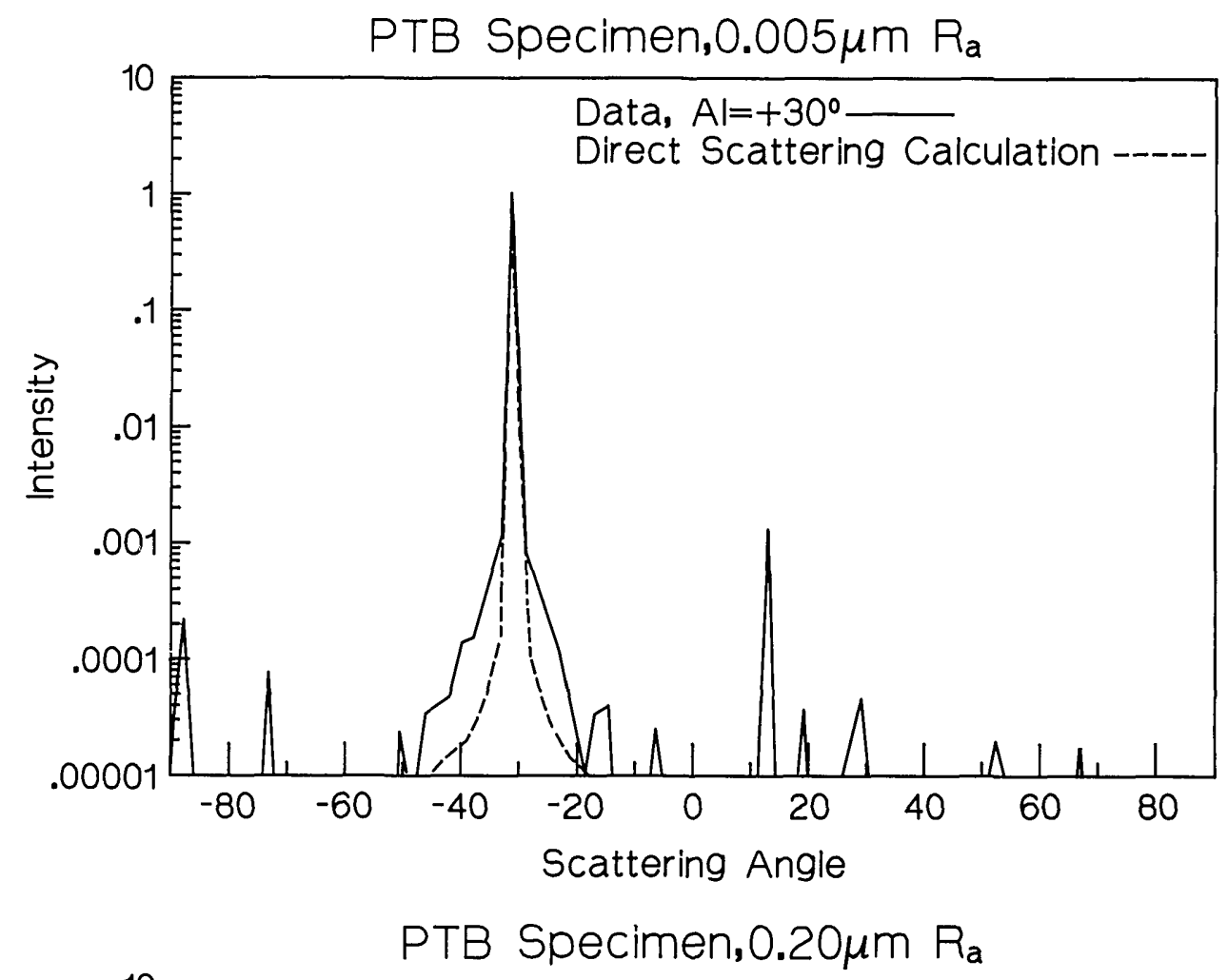

Figure 10-Data vs. calculation for the $R_{a}=0.20 \mu \mathrm{m}$ surface.

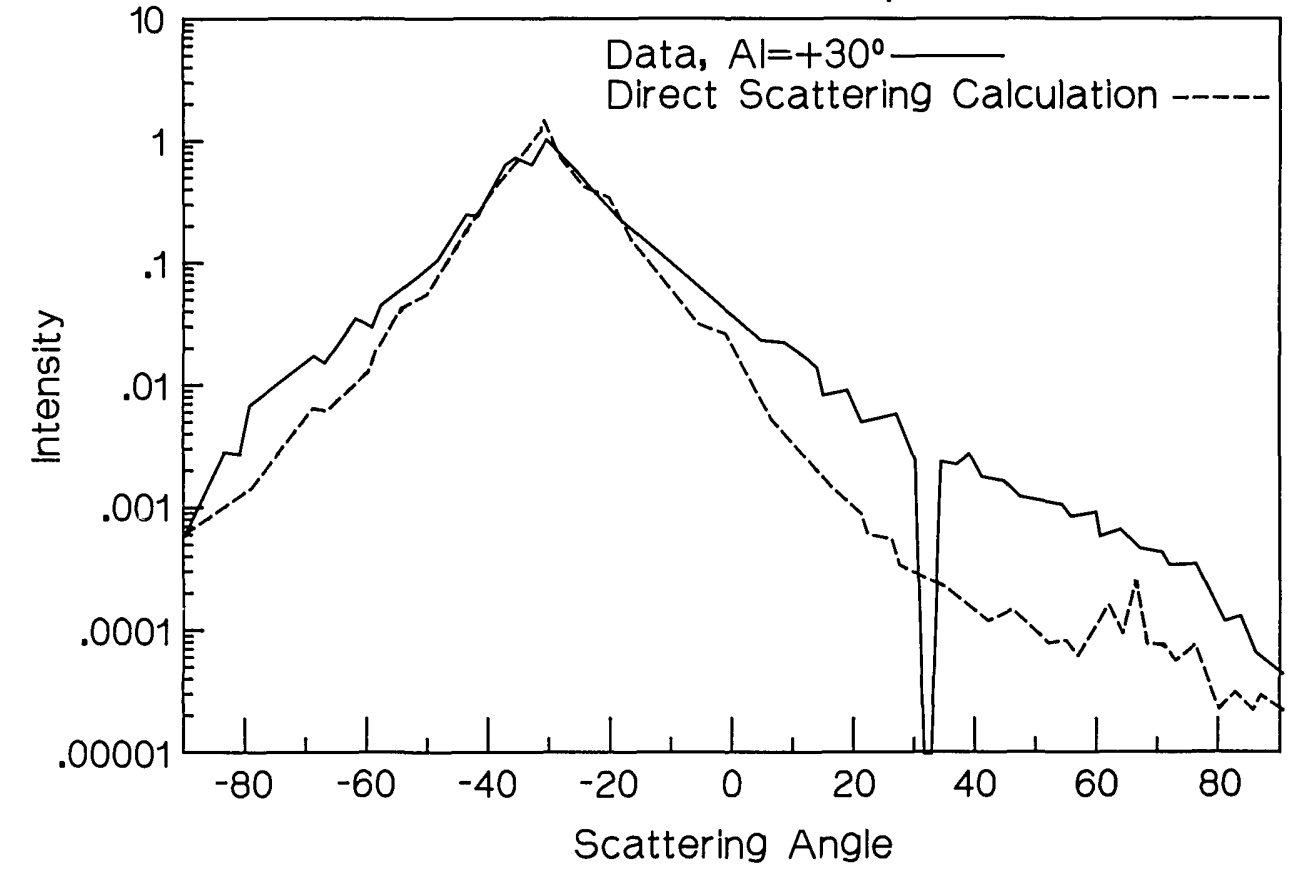

these approximations and observing how the agreement with the data is affected. We outline some of these possibilities below in terms of one experimental limitation and three model limitations:

1) It is possible that the stylus profiles should be taken with better horizontal resolution, i.e., there may be structures in the true surface profile with spatial wavelengths between 0.4 and $1.5 \mu \mathrm{m}$ that were not sufficiently resolved by the stylus instrument with its high spatial frequency cutoff of $1 \mu \mathrm{m}$. These structures may contribute significantly to the optical scattering. In fact, they would tend to increase the scattering on the outer wings, since short spatial wavelengths scatter light into large angles. 


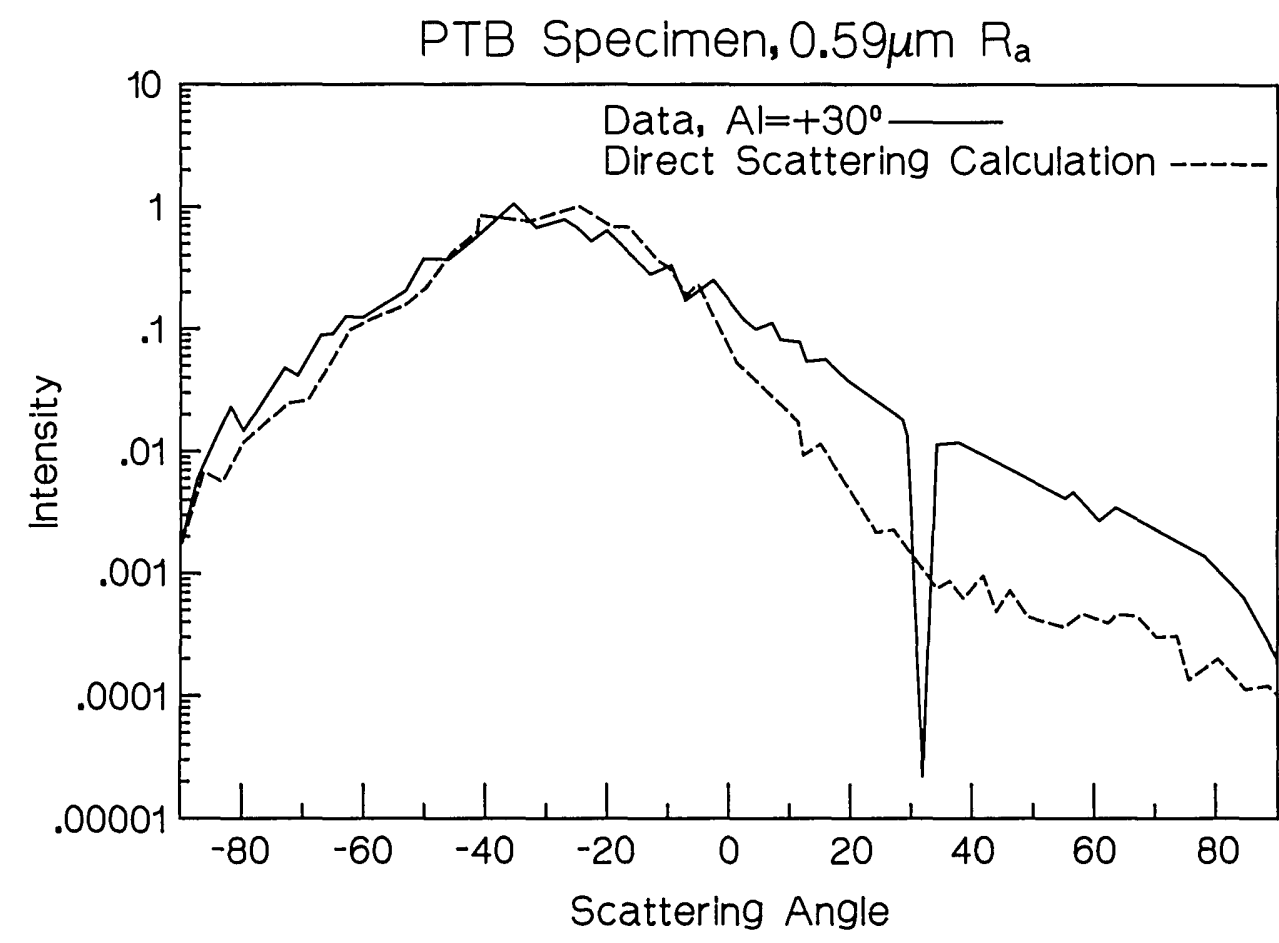

Figure 11-Data vs. calculation for the $R_{a}=0.59 \mu \mathrm{m}$ surface.

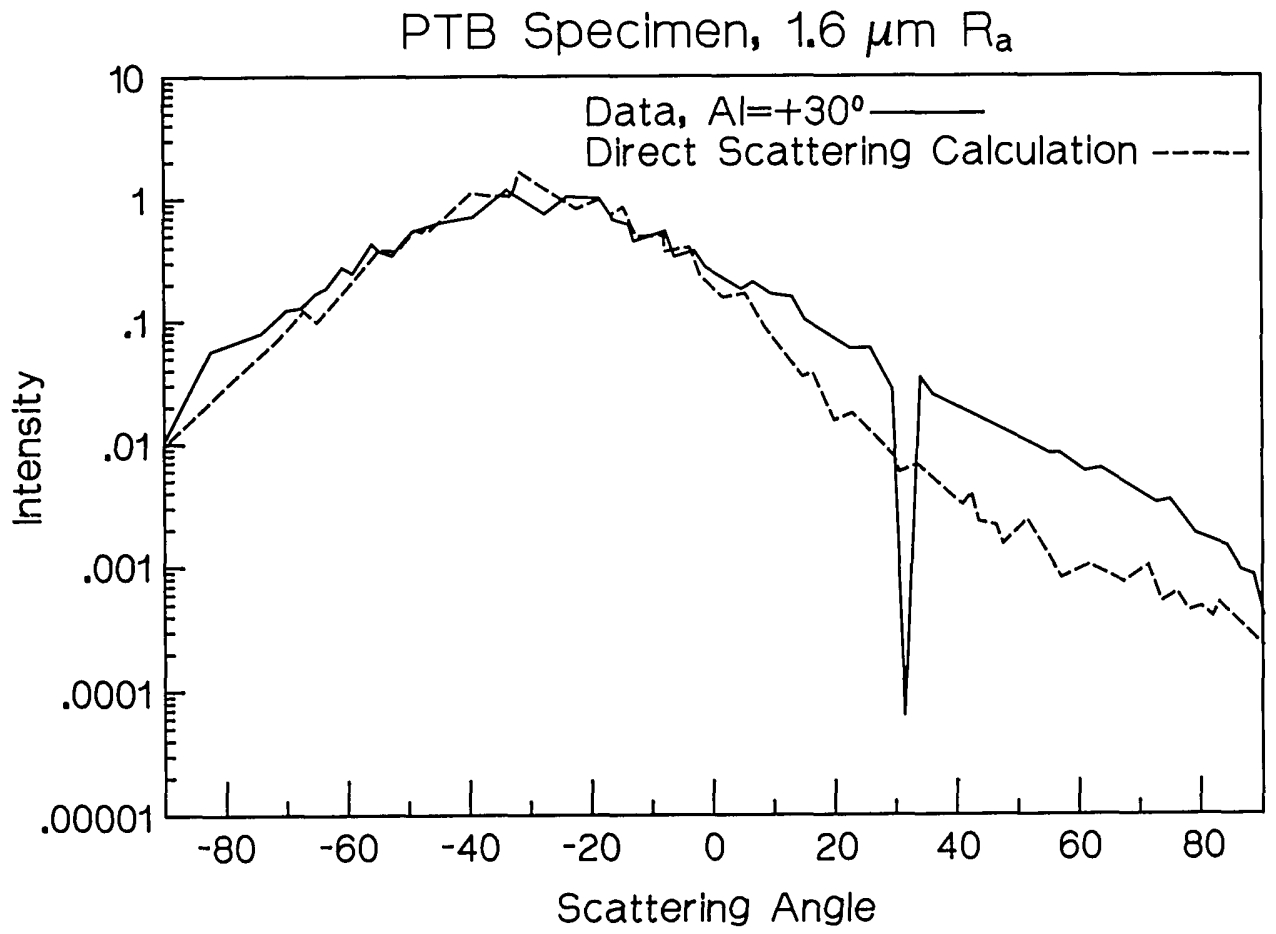

Figure 12-Data vs. calculation for the $R_{a}=1.6 \mu \mathrm{m}$ surface.

2) Equation (1) is the result of an integration by parts [21]. It neglects an additive contribution from the end points, 0 and $L$. This approximation seems valid provided the length $L$ is much greater than $\lambda$, but perhaps the approximation fails at low scattering intensities, where destructive interference due to phase cancellation effects in the scattering pattern is very high.

3) The preliminary analysis neglects any contribution from shadowing. It assumes that every point on the surface profile is illuminated with uniform intensity and contributes to the scattering at every 
angle $\theta_{s}$. However, it is likely that at grazing scattering angles, the outgoing wave from certain valleys is blocked by the peaks, and it is also possible that some of these valleys are shadowed from the incoming beam as well. The former effect probably tends to reduce the radiation scattered into the wings of the distribution, whereas the latter probably broadens the angular distribution by adding a degree of amplitude modulation to the already phase modulated outgoing wave.

4) It has been assumed in eq (1) that the electric field quantities on the surface are not functions of the local surface topography. This assumption implies several other assumptions, for example, that the reflection coefficient is not a function of either local slope or local curvature and that the electric field at each point on the surface is not affected by scattering from other points. All of these simplifications seem to be good ones for metallic surfaces where the reflection coefficients are fairly high, the surface slopes and curvatures small, and the significant roughness wavelengths much greater than $\lambda$. If some of the approximations were invalid, that might result in significant polarization effects in the angular scattering distributions. We have done experiments with linearly polarized light on the 0.59 and $0.20 \mu \mathrm{m}$ surfaces and have found no significant differences between the angular distributions for $s$ - and $p$-polarized incident beams, further suggesting that the simple theory may be valid for these surfaces. However, in view of the current differences between data and calculation, the breakdown of these simplifications and assumptions must be more carefully investigated, and more rigorous theories of electromagnetic scattering [22-23] should be applied to the roughness regime studied here.

\subsection{Previous Work}

Our experiment is a direct test of the BeckmannSpizzichino optical scattering theory for engineering surfaces where the roughness heights are the same order of magnitude as the wavelength of light. With the capabilities for measuring angular scattering distributions and storing and analyzing surface profiles, we have all the components for determining the level of complexity needed for a valid description of the optical scattering from these surfaces. Several previous studies on engineering surfaces [24-27] have correlated optical scattering measurements with roughness parameters such as $R_{a}$ or the rms roughness $R_{q}$ [19] obtained from stylus instruments, but they have not investigated the effects of the surface profiles directly. Chandley [28] and Thwaite [29] took a middle approach by comparing optical results with statistical functions generated from stylus profiles. Chandley compared the autocorrelation functions predicted from optical scattering distributions with those measured by stylus; Thwaite compared the power spectral densities calculated from stylus profiles with optical scattering distributions directly. However, both approaches involved theoretical assumptions that are not needed in the present work.

Experiments involving measured and calculated scattering distributions have been done for optical surfaces by Elson, Bennett, and Rahn [30,31]. Their work differs from ours in that the theory they used is more straightforward. Since the rms roughness $R_{q}$ was much less than $\lambda$ in the optical regime, first order Rayleigh theory could be used to analyze the optical scattering effects due to surface roughness. On the other hand, their experiments posed different kinds of difficulties from the present work. Optical surfaces generally produce low-intensity angular distributions that are strongly peaked in the forward direction near the specular beam, so the angular resolution and signal resolution requirements for their apparatus were high. Despite these differences, the agreement between theory and experiment for the previous studies is comparable to that observed here.

\subsection{Future Directions}

Our work is a preliminary step in the study of engineering surfaces by optical scattering. Once this direct scattering approach produces agreement with experiments for ideal, two-dimensional surfaces, its validity must be tested for real, anisotropic surfaces produced by many kinds of processes such as milling, grinding, and lapping. For these surfaces, there is a certain amount of light scattered slightly out of the plane of incidence, so the geometrical problem is only approximately two-dimensional. Highly isotropic surfaces such as those produced by shot blasting or electron-discharge machining must also be studied. In those cases, the scattering problem is truly threedimensional.

Finally, in order to use the optical scattering techiques for characterizing surfaces, comparisons with direct scattering methods are not sufficient. The inverse scattering problem must be solved adequately so that surface parameters such as $R_{q}$ may be derived in a reliable way solely from optical scattering data. This is where the speed and resulting economic benefits of on-line optical methods will be realized. 


\section{Appendix: Experimental Notes}

\subsection{Calibration}

The calibration of the 87 detectors is an important part of the operation of the apparatus. The relative sensitivity can vary by as much as a factor of 3 from one detector to the next. Therefore, at the beginning of each day's run, the system is calibrated in the following way. The specimen table is dropped below the center of the yoke, and a fixture with a flat mirror is inserted into the rotating mirror assembly. The surface of this mirror is located at the center of the yoke, but the mirror rotates with the M1, M2 assembly. This setup allows a laser beam of constant intensity to illuminate each of the detectors in turn as the mirror assembly is rotated. The 87 signals from the detector array are collected in this way and stored as a set of normalization data. The signals collected in the subsequent data runs are then normalized by dividing each detector reading by the corresponding normalization datum. The relative sensitivities of the detectors, when normalized in this way, are equal to within approximately $\pm 2 \%$ (1 standard deviation), a figure which includes the variation in sensitivity from one day to another.

In addition to the variation of sensitivity among the detector channels, there is an offset voltage signal at zero light level, which is constant with time but which varies from one detector to the next. Since the magnitudes of these offset signals are between 20 and $100 \mathrm{mV}$, and measurements are made which may be as small as $10 \mu \mathrm{V}$, these light-off signals must be subtracted from those measured with the light on. Therefore, each calibration run or data run actually consists of taking the difference between two scans of the detectors, a background scan measured with the laser beam diverted by a shutter, and a signal scan measured with the laser beam turned on.

\subsection{Stray Light}

A certain amount of stray light enters the detectors due to reflection from the ends of the optical fibers themselves. Approximately $1 \%$ of the light entering each lens is reflected from the fiber located at the focal point and refocused back to the surface. For a sharply peaked angular distribution, the effect influences the signals in the backscattered direction as shown in figure 13. The dotted line was taken under conditions which allow the light reflected from the detectors near $-30^{\circ}$, the specular direction, to propagate to the detectors located near the backscattering angle of $+30^{\circ}$. The solid line was taken by placing a dark mask to block the detectors near

\section{PTB Specimen, $0.20 \mu \mathrm{m} \mathrm{R}_{\mathrm{a}}, \mathrm{Al}=+30^{\circ}$}

Figure 13-Two angular distributions that show the effects of reflected stray light. The data shown by the solid line were taken with a dark shield masking the detectors around the specular beam when the backscattered detectors were scanned. The data represented by the dotted line were taken without masking and show a small shoulder in the backscattered direction.

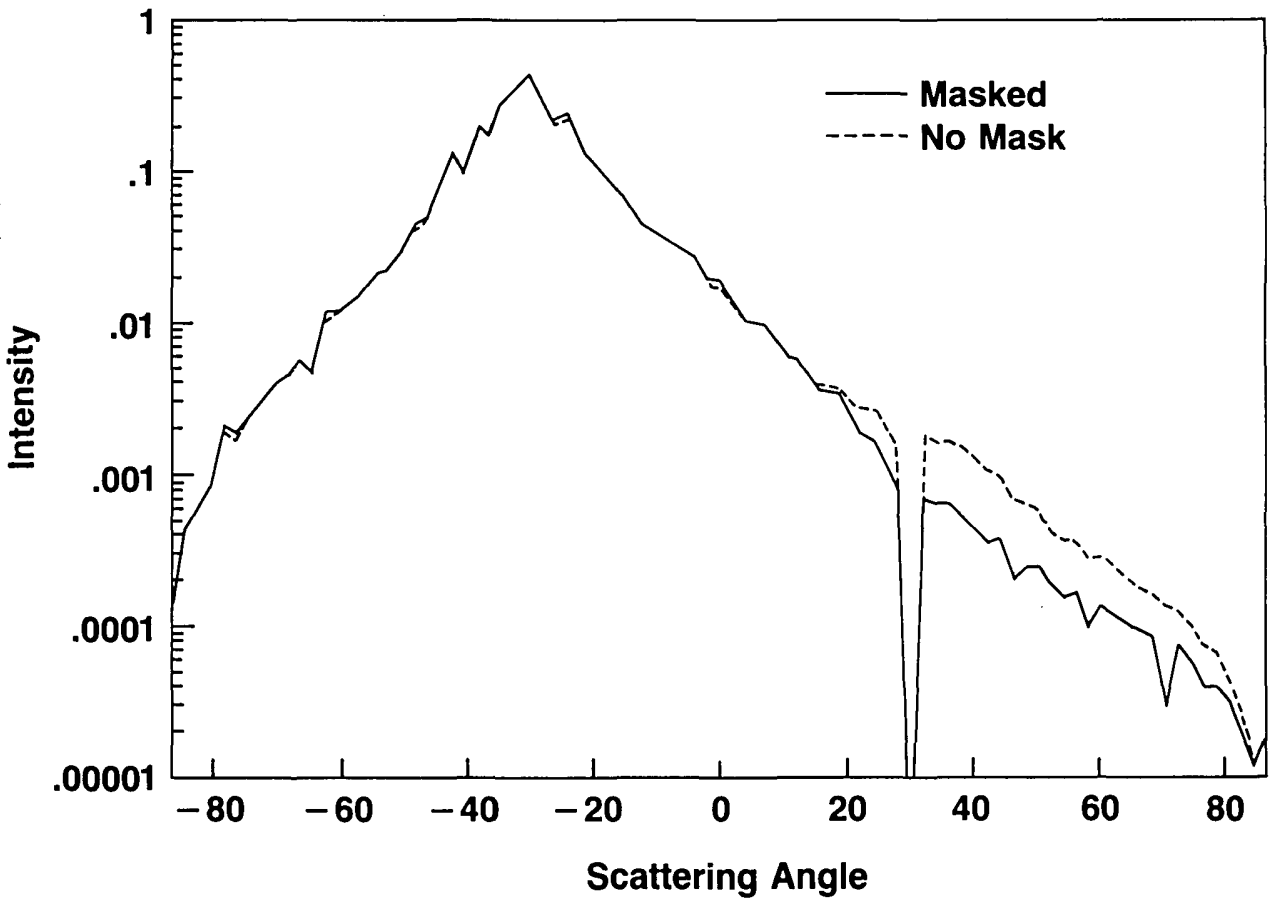


$-30^{\circ}$ as those near $+30^{\circ}$ were being scanned. The solid line represents a true angular distribution whereas the dotted line includes a shoulder around $+30^{\circ}$ due to the reflected stray light. All of the experimental distributions shown in figures 9-12 were measured with the masking approach. The difference between the curves of figure 13 is approximately a mirror image of the angular distribution itself but lower in intensity by about $21 / 2$ orders of magnitude.

It is important to note that this effect is significant only when the yoke is positioned vertically, when the angular distribution is sharply peaked, and when the surface is highly two-dimensional, so that most of the stray light is scattered by the surface back into the plane of incidence again.

In future experiments, we plan to model the effect of this reflected light on the detector signals and perform the appropriate subtractive massage on the measured angular distributions to correct for it.

The authors are grateful to K. M. Kunz, D. Medved, A. W. Hartman, J. Geist, E. F. Zalewski, and W. Gladden for key contributions during the construction and testing of DALLAS, and to members of the Fabrication Technology Division, particularly C. Summers and R. Lach, for the construction of a number of components. We would also like to thank S. A. Holliday for assistance during the early stages of the project, H. M. Helfer for photography of the apparatus and the angular distribution patterns, $S$. Morris for assistance in the preparation of the manuscript, and E. Marx, G. Blessing, and D. R. Flynn for valuable suggestions while the manuscript was in review.

\section{References}

[1] Gardner, C. S.; Alster, L. G. Electro-optic gauge for measuring the surface roughness of crankshaft bearings, presented at the 2nd Int. Prec. Eng. Seminar (National Bureau of Standards, Gaithersburg, MD, May 1983); Gardner, C. S.; Streight, W. E. U.S. Patent No. 4,364,663, $12 / 82$.

[2] Zombeck, M. V.; Wyman, C. C.; Weisskopf, M. C. High resolution x-ray scattering measurements for Advanced X-ray Astrophysics Facility (AXAF), in Radiation Scattering in Optical Systems. Proc. SPIE 257, 230 (1980).

[3] Vorburger, T. V.; Teague, E. C. Optical techniques for on-line measurement of surface topography. Prec. Eng. 3, 61 (1981).

[4] Corey, H. S. Surface finish from reflected laser light, in Advances in Optical Metrology. Proc. SPIE 153, 27 (1978).
[5] Teague, E. C.; Vorburger, T. V.; Maystre, D. M. Light scattering from manufactured surfaces. CIRP Annals 30, 563 (1981).

[6] Elson, J. M.; Bennett, J. M. Vector scattering theory, Opt. Eng. 18, 116 (1979).

[7] Church, E. L.; Zavada, J. M. Residual surface roughness of diamond-turned optics. Appl. Opt. 14, 1788 (1975).

[8] Stover, J. C.; Serati, S. A. Calculation of surface statistics from light scatter, in Precision Surface Metrology. Proc. SPIE 429, 96 (1983).

[9] Dainty, J. C., ed. Laser speckle and related phenomena, Topics in Applied Physics, Vol. 9 (Springer-Verlag, Berlin, 1975).

[10] Teague, E. C. Evaluation, revision, and application of the NBS stylus/computer system for the measurement of surface roughness. Natl. Bur. Stand. (U.S.) Tech. Note 902; 1976.

[11] Vorburger, T. V.; Teague, E. C.; Scire, F. E. Fast facility available for engineering needs. Dimensions/NBS 62, No. 11, 18 (Nov. 1978).

[12] Vorburger, T. V. FASTMENU: A set of FORTRAN programs for analyzing surface texture. Natl. Bur. Stand. (U.S.) NBSIR 83-2703; 1983.

[13] Similar specimens are discussed by Häsing, J., Herstellung und Eigenschaften von Referenznormalen für das Einstellen von Oberflächenmessgeräten. Werksattstechnik $\mathbf{5 5}, 380$ (1965).

[14] Beckmann, P.; Spizzichino, A. The Scattering of Electromagnetic Waves from Rough Surfaces (Pergamon, New York, 1963). Refs. 14,17,18 cite many other works dealing with the theory of optical scattering from rough surfaces.

[15] Azzam, R. M. A.; Bashara, N. M. Polarization characteristics of scattered radiation from a diffraction grating by ellipsometry with application to surface roughness. Phys. Rev. B5, 4721 (1972).

[16] Vorburger, T. V.; Ludema, K. C. Ellipsometry of rough surfaces, Appl. Opt. 19, 561 (1980).

[17] Bass, F. G.; Fuks, I. M. Wave Scattering from Statistically Rough Surfaces (Pergamon, London, 1979).

[18] Wirgin, A. Scattering from sinusoidal gratings: An evaluation of the Kirchoff approximation. J. Opt. Soc. Am. 73, 1028 (1983).

[19] American National Standards Institute B46.1-1978, Surface Texture (American Society of Mechanical Engineers, New York, 1978).

[20] Francon, M. Laser Speckle and Applications in Optics (Academic Press, New York, 1979), p.25.

[21] Ref. 14, p. 24f.

[22] Petit, R., ed. Electromagnetic theory of gratings, Topics in Current Physics, Vol. 22 (Springer-Verlag, Berlin, 1980).

[23] Maystre, D. Electromagnetic scattering from perfectly conducting rough surfaces in the resonance region. IEEE Trans. AP-31, 885 (1983).

[24] A few representative articles on light scattering experiments are given in refs. 4 and 25-31 below. A number of other articles are cited in ref. 3.

[25] Bennett, H. E.; Porteus, J. O. Relation between surface roughness and specular reflectance at normal incidence. $\mathbf{J}$. Opt. Soc. Am. 51, 123 (1961).

[26] Tanner, L. H. A comparison between Talysurf 10 and optical measurements of roughness and surface slope. Wear 57, 81 (1979).

[27] Smith, T. F.; Hering, R. G. Comparison of the Beckmann 
model with bidirectional reflectance measurements, presented at the ASME-AICH Heat Transfer Conf. (Atlanta, 1973), ASME publ. 73.HT-11.

[28] Chandley, P. J. Determination of the autocorrelation function of height on a rough surface from coherent light scattering. Opt. Quant. Elect. 8, 329 (1976).

[29] Thwaite, E. G. The direct measurement of the power spectrum of rough surfaces by optical Fourier transformation. Wear 57, 71 (1979), and A quantitative comparison of the wavelength spectrum of a surface obtained by optical Fourier transformation with calculations from profile measurements. Wear 83, 181 (1982).

[30] Elson, J. M.; Bennett, J. M. Relation between the angular dependence of scattering and the statistical properties of optical surfaces. J. Opt. Soc. Am. 69, 31 (1979).

[31] Elson, J. M.; Rahn, J. R.; Bennett, J. M. Light scattering from multilayer optics: comparison of theory and experiment. Appl. Opt. 19, 669 (1980). 